\title{
Descripción de biomecánica asociada a disyunción maxilar con anclaje esqueletal: Reporte de caso
}

\author{
Biomechanics description associated to bone anchored maxillary expander \\ treatment: A case report. \\ Patricio Rojas ${ }^{1 \mathrm{a}}$, Pedro Córdova ${ }^{1 \mathrm{a}}$, Geraldine Santos $^{1 \mathrm{a}}$, Carolina Vergara $^{1 \mathrm{a}}$
}

\section{RESUMEN}

Introducción: La discrepancia transversal es una anomalía común en ortodoncia, la cual se debe corregir tempranamente. Actualmente para su tratamiento existen disyuntores con anclaje dentario, híbrido o esqueletal, que limitarían los efectos adversos que han sido asociados a los disyuntores con anclaje dentario. Dada la falta de evidencia concreta sobre los efectos de la disyunción con anclaje esqueletal, es que este reporte profundiza en su biomecánica, determinando cambios dentarios y esqueletales, medidos en imágenes de tomografía computarizada cone-beam (TCCB).

Presentación clínica: Paciente de sexo masculino, 13 años de edad, con compresión maxilar de $6 \mathrm{~mm}$. Se trató con un disyuntor tipo BAME (bone-anchored maxillary expander), dispositivo soportado por 4 microtornillos palatales. Se tomaron registros de TCCB, previo (T0) y posterior a la disyunción (T1). Se registraron mediciones transversales a nivel anterior y posterior, modificando el protocolo de medición propuesto por Rojas et al. ${ }^{1}$. Se utilizaron puntos de referencias alveolares y dentarios, medidas angulares de inclinación de caninos y primeros molares, y se identificó el estadio de maduración de la sutura medio palatina (SMP), junto con la medición del diastema interincisivo.

Relevancia clínica: La expansión rápida maxilar con anclaje esqueletal constituye una herramienta terapéutica que se incorpora en los últimos años con la finalidad de aportar una expansión más efectiva en términos cuantitativos, disminuyendo los efectos adversos $^{2}$

Conclusión: El dispositivo BAME aumentó la dimensión transversal de tipo esqueletal evidenciado en los parámetros transverales alveolares y dentarios en una proporción similar, radiolucidez de la SMP, angulación molar invariable y disminución simétrica en la inclinación canina. Lo anterior demostró una disyunción maxilar paralela sin efectos biológicos adversos.
1. Universidad Andrés Bello, Viña del Mar, Chile.

a. Postgrado de Ortodoncia y Ortopedia Dentomaxilofacial

Correspondencia:

Carolina Vergara Santoro

Facultad de Odontología Universidad Andrés Bello, Viña del Mar-Chile Quillota 980, Viña del Mar carovsan@gmail.com

PALABRAS CLAVES

Técnica de expansión palatina; tomografía computarizada; cone beam; procedimientos de anclaje ortodóncico; hueso alveolar; reporte de caso.

KEYWORDS:

palatal expansion technique; cone beam; computed tomography; Orthodontic Anchorage Procedures; alveolar bone; case report. 


\section{ABSTRACT}

Introduction: Maxillary

transverse

discrepancy is a common anomaly in orthodontics, which should be corrected early in life. There are currently tooth-anchored, hybrid, or skeletal anchorage disjunctors available for its treatment, limiting the adverse effects associated with toothanchored disjunctors. Given the lack of concrete evidence on the effects of disjunction with skeletal anchorage, this report delves into its biomechanics, determining dental and skeletal changes measured on cone-beam computed tomography (CBCT) images.

Clinical case: A 13-years old male patient with a $6 \mathrm{~mm}$ maxillary compression. It was treated with a BAME expander (boneanchored maxillary expander), a device supported by four palatal micro screws. CBCT records were taken, before (T0) and after disjunction (T1), in addition to a control to evaluate the positioning of the micro screws. Transverse measurements were recorded at the anterior and posterior levels, modifying the measurement protocol proposed by Rojas et al.1. In addition, alveolar and dental reference points, angular measurements of canine, and first molar inclination were used, and the stage of maturation of the midpalatal suture (MPS) was identified, together with the measurement of the interincisive diastema.

\section{Clinical relevance: Rapid maxillary} expansion with skeletal anchorage is a therapeutic tool incorporated in recent years to provide a more effective expansion in quantitative terms, reducing adverse effects ${ }^{2}$.

Conclusion: The BAME device increased the skeletal transverse dimension, as evidenced in the alveolar and dental transverse parameters in a similar proportion, SMP radiolucency, unchanged molar angulation, and symmetrical decrease in canine inclination. All the above showed a parallel maxillary disjunction without adverse biological effects.

\section{INTRODUCCIÓN}

Los tratamientos de expansión maxilar han evolucionado desde la introducción del anclaje esqueletal, el cual limitaría los efectos adversos que han sido asociados a los disyuntores con anclaje dentario exclusivo. Esta técnica fue presentada por Winsauer et al. ${ }^{2}$ con el expansor Micro 4 y Micro 6. El primero con 4 microtornillos palatinos, utilizado en pacientes adolescentes, y el segundo con 6 microtornillos, indicado en pacientes adultos. Posteriormente Lee et al. $^{3}$ pone en evidencia los efectos de este expansor, bajo un modelo de análisis tridimensional de elementos finitos donde explica la biomecánica asociada al estrés que genera la expansión maxilar. Entre los beneficios que se plantearon se encuentran la ausencia de efectos dentoalveolares indeseados, como la reabsorción radicular y el tipping o inclinación dentaria ${ }^{2}$.

Al comparar los efectos de los disyuntores con anclaje dentario y esqueletal, la bibliografía actual no es concluyente. Existe evidencia en la que se observan diferencias entre ambas técnicas, produciéndose una expansión mas bien paralela con un movimiento más esquelético que dentario en los disyuntores con anclaje esqueletal $^{1}$, como también existen estudios que muestran que los cambios dentarios y esqueletales son similares para ambos tipos de aparatos $^{4}$.

Ante la falta de evidencia concreta con respecto a los efectos de la disyunción con anclaje esqueletal exclusivo, el siguiente reporte de caso expone y detalla la biomecánica asociada a su uso, determinando los cambios esqueletales y dentarios, medidos en imágenes de TCCB.

\section{CASO CLÍNICO}

Paciente sexo masculino, 13 años, sin antecedentes sistémicos relevantes, ingresa como paciente del Programa de Especialización en Ortodoncia y Ortopedia Dentomaxilofacial de la Universidad Andrés Bello, Viña del Mar, Chile, donde su atención cumplió con todas las normas éticas correspondientes, considerando la firma del consentimiento informado. 
Dentro del diagnóstico, dentoalveolarmente presenta una compresión maxilar de $6 \mathrm{~mm}$, medida mediante el análisis Wala-Ridge y un estado de maduración ósea cervical CS4 según el método propuesto por Baccetti et al. ${ }^{5}$.
En su plan de tratamiento se consideró realizar una expansión rápida maxilar con anclaje esqueletal, la cual se llevó a cabotravés de un dispositivo tipo BAME que incorporó un tornillo

Figura I: Registros fotográficos intraorales, pre y post-instalación de microtornillos, post instalación de BAME, y post disyunción.

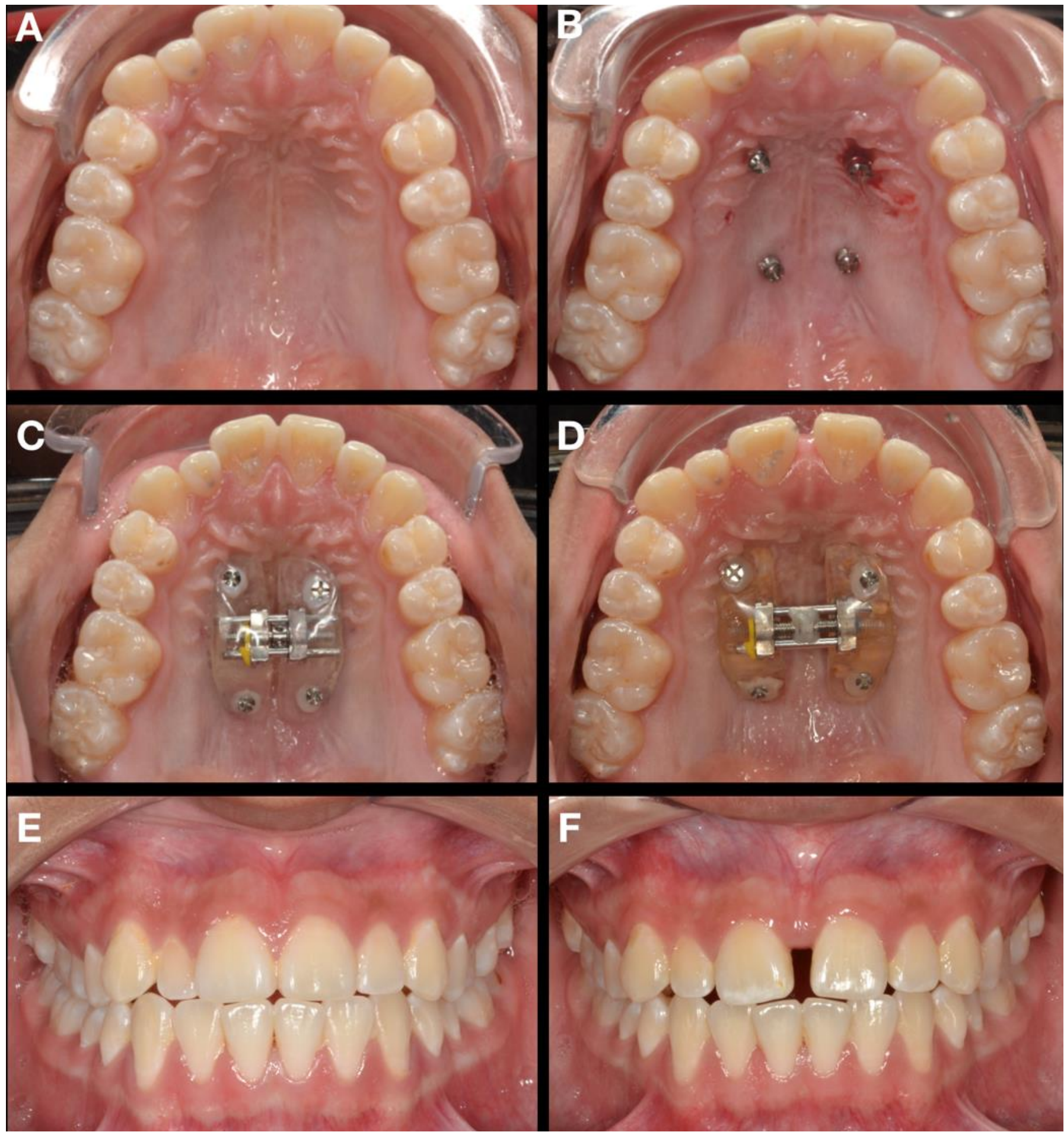

(A) Fotografía inicial previa a tratamiento. (B) Fotografía posterior a colocación de microtornillos. (C) Fotografía posterior a colocación de dispositivo tipo BAME sin activación. (D) Fotografía post-disyunción con apertura de tornillo central de $10 \mathrm{~mm}$ y cierre de tornillo central con entorche de alambre de tarno y resina flow. E: Fotografía frontal previa a tratamiento. F: Fotografía frontal post-disyunción. 
de expansión de $11 \mathrm{~mm}$ marca Morelli (Sorocaba, São Paulo, Brasil). Este dispositivo fue soportado por 4 microtornillos marca Jeil Medical (Jeil Medical Corporation, Seul, Republic of Korea), modelo JS. Dos microtornillos anteriores (entre canino y primer premolar) de $12 \mathrm{~mm}$ de longitud y $\varnothing$ $2.0 \mathrm{~mm}$ y dos posteriores (entre segundo premolar y primer molar) de $8 \mathrm{~mm}$ de longitud y $\varnothing 1.6 \mathrm{~mm}$.

La expansión se llevó a cabo con el protocolo de $1 / 4$ vuelta diaria $(0.25 \mathrm{~mm})$, durante 40 días, tiempo necesario hasta normalizar la dimensión transversal. La cantidad de apertura del tornillo de expansión fue de $10 \mathrm{~mm}$ considerando la sobrecorrección (Figura I).

Tabla 1: Protocolo de medición de expansión maxilar con anclaje esqueletal, a través TCCB, modificado de Roias et al.

\begin{tabular}{|c|c|c|c|}
\hline Parámetro & Plano de Corte & Referencia de corte & Definición operacional \\
\hline $\begin{array}{l}\text { Dimensión transversal } \\
\text { anterior }\end{array}$ & Axial & $\begin{array}{l}\text { Furca del 1er molar superior } \\
\text { D-I }\end{array}$ & $\begin{array}{l}\text { Medida lineal entre la cortical vestibular derecha e izquierda, teniendo } \\
\text { como referencia anteroposterior el punto medio en la cortical vestibular } \\
\text { entre canino superior y ler premolar superior. }\end{array}$ \\
\hline $\begin{array}{l}\text { Dimensión transversal } \\
\text { posterior }\end{array}$ & Axial & $\begin{array}{l}\text { Furca del 1er molar superior } \\
\text { D-I }\end{array}$ & $\begin{array}{l}\text { Medida lineal entre la cortical vestibular derecha e izquierda, teniendo } \\
\text { como referencia anteroposterior el punto medio de la cortical vestibular } \\
\text { entre el } 2 \text { do premolar superior y el 1er molar. }\end{array}$ \\
\hline $\begin{array}{l}\text { Inclinación molar } \\
(\mathrm{D}-\mathrm{I}) *\end{array}$ & Coronal & $\begin{array}{l}\text { Corte tangente a la unión del } \\
\text { centro de las coronas de los } \\
\text { caninos D-I }\end{array}$ & $\begin{array}{l}\text { En el plano coronal el ángulo formado por la referencia horizontal del } \\
\text { software y un plano de referencia molar (PRM). El PRM fue trazado } \\
\text { del centro del ancho del paladar a nivel del piso palatino y un punto en } \\
\text { el 1er molar superior ubicado en la cúspide palatina. }\end{array}$ \\
\hline
\end{tabular}

\begin{tabular}{|c|c|c|}
\hline $\begin{array}{l}\text { Inclinación canina } \\
(\mathrm{D}-\mathrm{I})\end{array}$ & Coronal & $\begin{array}{l}\text { Corte tangente a la unión de } \\
\text { la cúspide del canino superior } \\
\text { D-I }\end{array}$ \\
\hline
\end{tabular}

En el plano coronal el ángulo formado por la referencia horizontal del software y un plano de referencia canino (PRC). El PRC fue trazado del centro del ancho del paladar a nivel del piso palatino y la cúspide del canino superior.

\begin{tabular}{|c|c|c|c|}
\hline $\begin{array}{l}\text { Longitud Ápice - Ápice } \\
\text { canino (D - I) }\end{array}$ & Axial & $\begin{array}{l}\text { Corte tangente a la unión de } \\
\text { los ápices del canino superior } \\
\text { D-I }\end{array}$ & Longitud medida entre ápices de canino superior derecho e izquierdo. \\
\hline $\begin{array}{l}\text { Longitud Ápice - Ápice } \\
\text { Mesio-Vestibular } 1 \text { er } \\
\text { molar (D - I) }\end{array}$ & Axial & $\begin{array}{l}\text { Corte tangente a la unión de } \\
\text { los ápices de la raíz palatina } \\
\text { de los 1eros molares } \\
\text { superiores D-I }\end{array}$ & $\begin{array}{l}\text { Longitud medida entre ápices de la raíz mesiovestibular del 1er molar } \\
\text { superior derecho e izquierdo. }\end{array}$ \\
\hline
\end{tabular}

\begin{tabular}{llll}
\hline Longitud & cara & Corte tangente al punto más & \\
Vestibular - Vestibular & Axial & convexo de las coronas de los & Longitud medida entre la superficie vestibular en su punto más externo \\
canino (D-I) & caninos superiores D-I & de la corona del canino superior derecho e izquierdo.
\end{tabular}

Longitud cara
Vestibular - Vestibular Axial
1er molar (D-I)

Corte tangente al punto más

convexo de las coronas de los Longitud medida entre la superficie vestibular en su punto más externo, 1ros molares superiores D-I a nivel de la cúspide mesio-vestibular de la corona del 1er molar Respecto a la cúspide Mesio- $\quad$ superior derecho e izquierdo 
Se solicitó TCCB de maxilar superior en tres tiempos: pre-disyunción (T0), control de posicionamiento de microtornillos y postdisyunción (T1). Los registros de TCCB fueron capturados con el mismo tomógrafo (Gendex CB500, Imaging Sciences International LCC, Hatfield PA, USA) con los siguientes parámetros: campo de visión 80x80 mm Ø, 60-90 kV, 1-14mA y tamaño de vóxel de $200 \mu \mathrm{m}$. Posteriormente, los datos fueron almacenados en formato Digital Imaging and Communication in Medicine (DICOM), los cuales fueron exportados y visualizados en el software Horos ${ }^{\mathrm{TM}}$ versión 3.3.5 (The Horos Project), en un equipo Macbook Pro (MacOS 10.14 Mojave, Pantalla LED 13.3 pulgadas, resolución 1280x800 pixeles, compatible con millones de colores, gráficos Intel HD Graphics 40001536 MB). Durante las tomas se pidió al paciente que permaneciera de pie, plano sagital perpendicular al piso y plano de Frankfort paralelo al piso.

En la literatura no hay consenso para cuantificar la disyunción maxilar a través de TCCB, puesto que hay diferentes volúmenes de captura y se proponen diferentes puntos de referencia. Por lo anterior, las medidas se realizaron adaptando el protocolo de medición propuesto por Rojas et al. ${ }^{1}$ (Tabla I), el cual presenta en su estudio volúmenes de captura similares, además de puntos de referencia replicables, el cual fue aplicado en este estudio (Figura II). Las medidas del reportadas en este caso fueron registradas por una investigadora especialista en ortodoncia y ortopedia dentomaxilofacial (Tabla II)

Tabla II: Medidas Pre (T0) y Post (T1) disyunción con anclaje esquelético medido a través de TCCB de reconstrucción multiplanar

\begin{tabular}{|c|c|c|c|c|}
\hline Parámetro & $\begin{array}{l}\text { Plano de } \\
\text { Corte }\end{array}$ & T1 & $\mathbf{T} 2$ & Diferencia \\
\hline $\begin{array}{l}\text { Dimensión transversal } \\
\text { anterior }\end{array}$ & Axial & $3.939 \mathrm{~cm}$ & $4.310 \mathrm{~cm}$ & $+0.371 \mathrm{~cm}(3.71 \mathrm{~mm})$ \\
\hline $\begin{array}{l}\text { Dimensión transversal } \\
\text { posterior }\end{array}$ & Axial & $5.164 \mathrm{~cm}$ & $5.461 \mathrm{~cm}$ & $+0.297 \mathrm{~cm}(2.97 \mathrm{~mm})$ \\
\hline $\begin{array}{l}\text { Inclinación molar } \\
(\mathrm{D}-\mathrm{I})^{*}\end{array}$ & Coronal & $\begin{array}{l}\text { D: } 46.341^{\circ} \\
\text { I: } 47.317^{\circ}\end{array}$ & $\begin{array}{l}\text { D: } 46.025^{\circ} \\
\text { I: } 46.471^{\circ}\end{array}$ & $\begin{array}{l}-0.316^{\circ} \\
-0.846^{\circ}\end{array}$ \\
\hline $\begin{array}{l}\text { Inclinación canina } \\
(\mathrm{D}-\mathrm{I})\end{array}$ & Coronal & $\begin{array}{l}\text { D: } 24.905^{\circ} \\
\text { I: } 26.637^{\circ}\end{array}$ & $\begin{array}{l}\text { D: } 21.730^{\circ} \\
\text { I: } 22.992^{\circ}\end{array}$ & $\begin{array}{l}-3.175^{\circ} \\
-3.645^{\circ}\end{array}$ \\
\hline $\begin{array}{l}\text { Longitud Ápice - Ápice } \\
\text { canino (D - I) }\end{array}$ & Axial & $2.647 \mathrm{~cm}$ & $2.950 \mathrm{~cm}$ & $+0.303 \mathrm{~cm}(3.03 \mathrm{~mm})$ \\
\hline $\begin{array}{l}\text { Longitud Ápice }- \text { Ápice } \\
\text { Mesio-Vestibular 1er molar } \\
\text { (D - I) }\end{array}$ & Axial & $4.989 \mathrm{~cm}$ & $5.253 \mathrm{~cm}$ & $+0.264 \mathrm{~cm}(2.64 \mathrm{~mm})$ \\
\hline $\begin{array}{l}\text { Longitud cara Vestibular - } \\
\text { Vestibular canino (D-I) }\end{array}$ & Axial & $3.653 \mathrm{~cm}$ & $3.962 \mathrm{~cm}$ & $+0.309 \mathrm{~cm}(3.09 \mathrm{~mm})$ \\
\hline $\begin{array}{l}\text { Longitud cara Vestibular - } \\
\text { Vestibular 1er molar (D-I) }\end{array}$ & Axial & $5.302 \mathrm{~cm}$ & $5.600 \mathrm{~cm}$ & $+0.298 \mathrm{~cm}(2.98 \mathrm{~mm})$ \\
\hline
\end{tabular}


Figura II: Mediciones Pre y post tratamiento en TCCB.
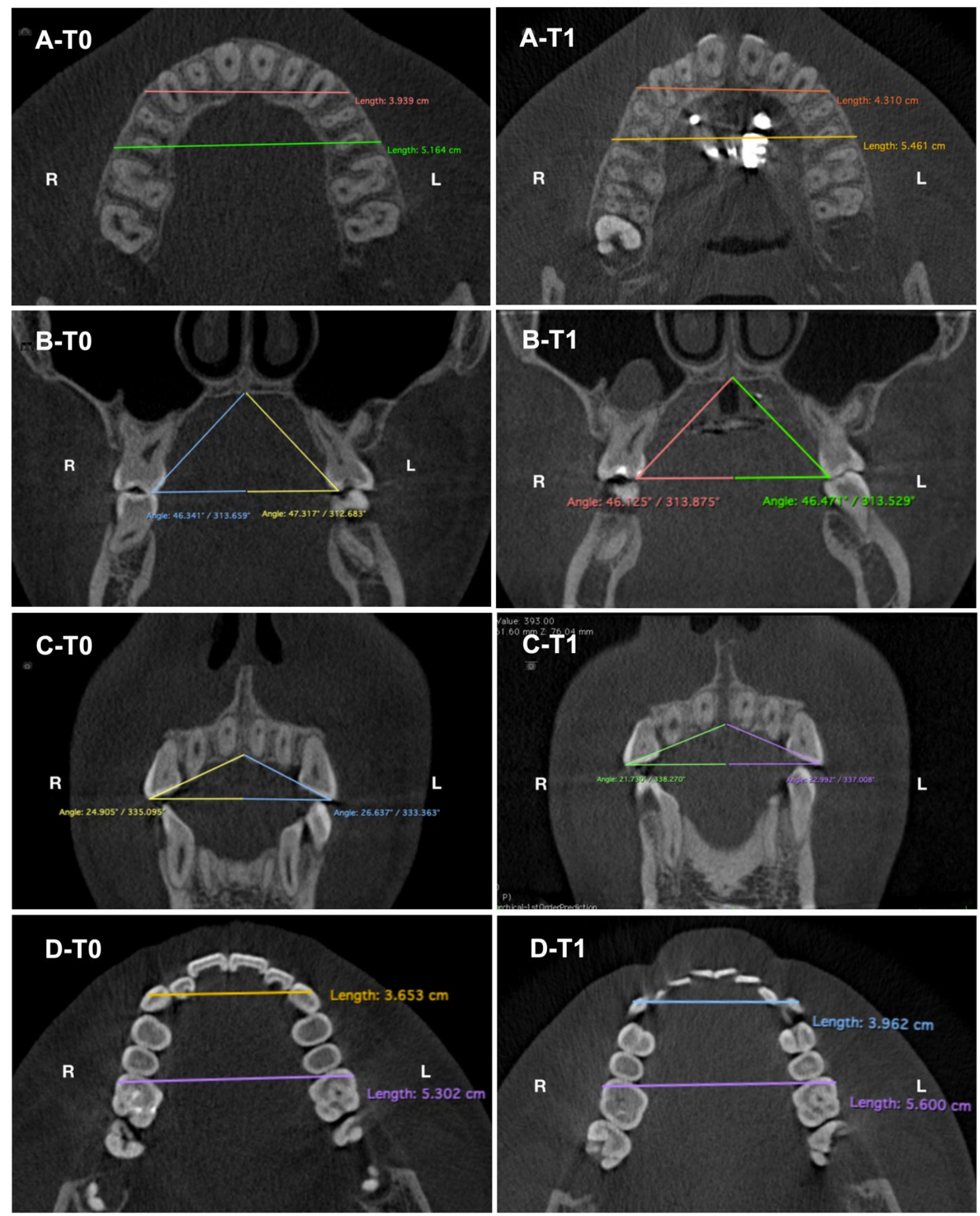

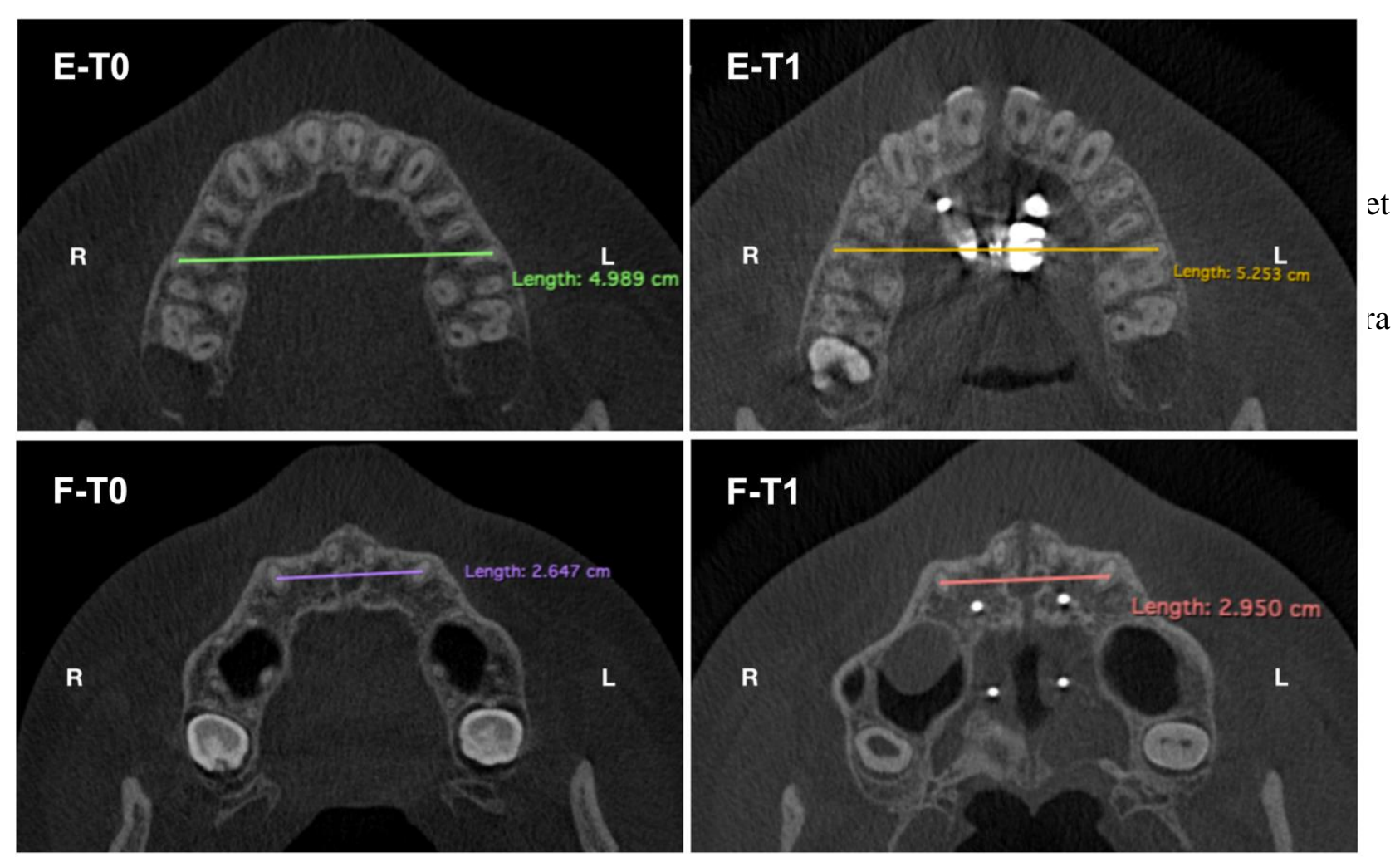

(A) Determinación de Dimensión transversal anterior y posterior. (B) Determinación de angulación molar. (C) Determinación de angulación canina. (D) Determinación de longitud vestibular-vestibular a nivel de canino y primer molar. (E) Determinación longitud ápice-ápice a nivel de primer molar. $(F)$ Determinación longitud ápice-ápice a nivel de caninos. (T0) Medicion pretratamiento. (T1) Medición post tratamiento.

\section{RELEVANCIA CLÍNICA}

La expansión rápida maxilar con anclaje esqueletal constituye una herramienta terapéutica que se ha incorporado en los últimos años con la finalidad de aportar una expansión más efectiva en términos cuantitativos, disminuyendo los efectos $\operatorname{adversos}^{2}$.

En este reporte, las mediciones transversales lineales mostraron una expansión más bien paralela, concordando con lo reportado por Lin et al. ${ }^{6}$. El paciente experimentó un aumento en la dimensión transversal anterior y posterior, el que mostró un incremento de $3.71 \mathrm{~mm}$ a nivel anterior y $2.97 \mathrm{~mm}$ a nivel posterior, asociado a una apertura de $10 \mathrm{~mm}$ del tornillo central. Rojas et al. ${ }^{1}$ encontró resultados similares, donde con dispositivos BAME consiguió en promedio un aumento en la dimensión transversal anterior de $3 \mathrm{~mm}$ y de $2.4 \mathrm{~mm}$ a nivel posterior, con una activación de $7.33 \mathrm{~mm}$.
La variación en el diseño, el sitio de anclaje, la distribución de tensiones y el patrón de expansión, carresponden a variables que determinan el resultado de la expansión ${ }^{6}$.

Respecto a la inclinación molar, esta permaneció prácticamente invariable. Resultados similares se pueden encontrar ya desde el 2008, donde Iida et al. $^{8}$ sostiene que el anclaje esqueletal resuelve el problema de estrés en piezas dentarias al no usarlas como anclaje, y donde el propio autor registra nula inclinación molar en su reporte. Resultados similares fueron reportados por Vanarsdall et al. ${ }^{9}$, Winsauer et al. ${ }^{2}$ y Mosleh et al. ${ }^{10}$, sin embargo, se contrapone a los resultados de Lagravère et al. $^{4}$, inesperados incluso para el propio autor, quien encontró un aumento de igual magnitud en la inclinación molar a los observados en dispositivos de anclaje entario. 
Por otro lado, el estudio de Rojas et al. ${ }^{1}$ reportó también un aumento de angulación molar, aunque de menor magnitud, al ser comparados con dispositivos de anclaje dentario.

Si bien la evidencia aún no es concluyente, pareciera haber cierta tendencia a que los dispositivos tipo BAME generan pocos cambios en la inclinación molar.

Diversos autores han indicado que el centro de rotación en el plano frontal para la disyunción con anclaje dentario es único y se encuentra cercano a la sutura frontonasal ${ }^{11,12}$, esto permite que ambos segmentos del maxilar se separen en un movimiento piramidal causando mayor inclinación a nivel dentoalveolar.

Cantarella et al. ${ }^{13}$ analizó el centro de rotación de un disyuntor con anclaje esqueletal MSE, en el cual observa que la relación entre el hueso maxilar y cigomático se mantiene durante la expansión, rotando juntos alrededor de un centro de rotación común, el cual se concluye se encuentra ligeramente superior a la sutura frontocigomática. Esto permite inferir que existen dos centros de resistencia, uno para cada complejo cigomáticomaxilar, lo cual explicaría el movimiento más paralelo de la sutura en el plano frontal, disminuyendo la inclinación molar, lo cual se evidencia en este reporte de caso. Aún así es importante indicar que, a pesar de esto, se puede reportar un grado de inclinación en los molares, debido a que este centro de rotación no necesariamente se encuentra al mismo nivel del complejo dentoalveolar, efecto observado en los resultados de Lagravère et al. ${ }^{4}$ y Rojas et al. ${ }^{1}$.

En el presente caso se constató en T1 la presencia de radiolucidez en la SMP y diastema interincisivo de $2.75 \mathrm{~mm}$ equivalente al $\approx 28 \%$ de la apertura del tornillo del dispositivo BAME. Resultados relativamente inferiores a lo reportado por Rojas et al. ${ }^{1}$ quien reportó un diastema promedio de $2.54 \mathrm{~mm}$ equivalente a un $36 \%$ de la apertura del tornillo.
Finalmente se observó una disminución simétrica de la inclinación canina $\left(\approx 3^{\circ}\right)$, lo que podría deberse a una compensación natural del canino en busca de acople dentario, producto de la acción muscular de las mejillas.

Los dispositivos de anclaje esqueletal en general no están libres de inconvenientes, así lo describe Iida et al. ${ }^{8}$ quien menciona el alto costo y la menor estabilidad durante la consolidación de la sutura, esto porque atribuye la existencia de procesos inflamatorios de la mucosa palatina alrededor de los microtornillos, que pudiesen en alguna medida generar pérdida de activación del tornillo central. Esto no fue constatado puesto que al igual que propone Iida et al. ${ }^{8}$ el cierre del tornillo con resina flow en el tornillo central y en el propio caso clínico, de entorchar con alambre de tarno, propició la no pérdida de activación. Por otro lado, se han reportado complicaciones que incluyen disyunción asimétrica lo que trae como consecuencia una oclusión inestable y división desfavorable del tabique interradicular entre los incisivos centrales, lo que pone al paciente en alto riesgo periodontal a largo plazo, con formación de sacos periodontales y recesiones gingivales ${ }^{14}$, complicaciones no reportadas en este reporte de caso.

Desde la otra arista, es relevante mencionar que aparte de las ventajas mecánicas que suponen los dispositivos tipo BAME, existen ciertas ventajas clínicas. La literatura indica que este tipo de dispositivos puede ser muy útil en pacientes con ausencia de dientes posteriores o en aquellos donde la salud de éstos se encuentra comprometida, permitiendo la realización de la terapia de ortodoncia al mismo tiempo que la expansión, acortando así los tiempos de tratamiento. Además, facilita una mejor higiene bucal dado su tamaño y diseño más pequeños ${ }^{15}$.

A pesar de las limitaciones por ser un reporte de caso, este artículo da información relevante respecto a la biomecánica asociada a la disyunción con anclaje esqueletal. Se sugiere realizar estudios observacionales y analíticos que puedan dar respuesta con mayor nivel de evidencia. 
En conclusión, el dispositivo tipo BAME aplicado generó un aumento en la dimensión transversal de tipo esqueletal evidenciado en los parámetros transverales alveolares y dentarios en similar proporción, radiolucidez de la SMP, angulación molar invariable y disminución simétrica en la inclinación canina. Lo anterior demostró una disyunción maxilar paralela sin efectos biológicos adversos

\section{CONFLICTOS DE INTERÉS}

Los autores declaran no presentar conflictos de interés

\section{REFERENCIAS}

[1] Rojas V, Macherone C, Zursiedel MI, Valenzuela JG. Rapid maxilary expansion in young adults: comparison of tooth-borne and bone-borne appliances, a cohort study. J Oral Res. 2019;8(3):201-9.

DOI:10.17126/joralres.2019.031.

[2] Winsauer $\mathrm{H}$, Vlachojannis $\mathrm{J}$, Winsauer C, Ludwig B, Walter A. A Bone-Borne appliance for rapid maxillary expansion. J Clin Orthod. 2013;47(6):375-81.

[3] Lee SC, Park JH, Bayome M, Kim KB, Araujo EA, Kook YA. Effect of boneborne rapid maxillary expanders with and without surgical assistance on the craniofacial structures using finite element analysis. Am J Orthod Dentofac Orthop. 2014;145(5):638-48. DOI:10.1016/j.ajodo.2013.12.029.

[4] Lagravère MO, Carey J, Heo G, Toogood RW, Major PG. Transverse, vertical, and anteroposterior changes from bone-anchored maxillary expansion vs traditional rapid maxillary expansion: A randomized clinical trial. Am J Orthod Dentofac Orthop. 2010;137(3):304.e1-12.

DOI:10.1016/j.ajodo.2009.09.016.

[5] Baccetti T, Franchi L, McNamara JA Jr. The cervical vertebral maturation (CVM) method for the assessment of optimal treatment timing in dentofacial orthopedics. Semin Orthod. 2005;1;11(3):119-29.

DOI:10.1053/j.sodo.2005.04.005
[6] Lin L, Ahn HW, Kim SJ, Moon SC, Kim $\mathrm{SH}$, Nelson G. Tooth-borne vs boneborne rapid maxillary expanders in late adolescence. Angle Orthod. 2015;85(2): 253-62.

DOI: 10.2319/030514-156.1.

[7] Bazargani F, Feldmann I, Bondemark L. Three-dimensional analysis of effects of rapid maxillary expansion on facial sutures and bones: a systematic review. Angle Orthod. 2013;83(6):1074-1082.

DOI: 10.2319/020413-103.1.

[8] Lida S, Haraguchi S, Aikawa T, Yashiro K, Okura M, Kogo M. Conventional boneanchored palatal distractor using an orthodontic palatal expander for the transverse maxillary distraction osteogenesis. Oral Surg Oral Med Oral Pathol Oral Radiol Endod. 2008;105(2): e8-e11.

DOI:10.1016/j.tripleo.2007.08.022.

[9] Vanarsdall RL. Jr, Secci AG. Periodontal - Orthodontic interrelationships. In: Graber LW, Vanarsdall RL, Vig KWL. Orthodontic: Current Principles and Techniques. Philadelphia: Elsevier/Mosby; 2011. 807-42.

[10] Mosleh MI, Kaddah MA, Abd-ElSayed FA, EISayed HS. Comparison of transverse changes during maxillary expansion with 4-point bone-borne and tooth-borne maxillary expanders. Am. J. Orthod. Dentofac. Orthop., 148(4):599-607, 2015. DOI:10.1016/j.ajodo.2015.04.040.

[11] Braun S, Bottrel JA, Lee KG, Lunazzi JJ, Legan HL. The biomechanics of rapid maxillary sutural expansion. Am J Orthod Dentofac Orthop. 2000;118(3): 257-61. DOI:10.1067/mod.2000.108254.

[12] Jafari A, Shetty KS, Kumar M. Study of stress distribution and displacement of various craniofacial structures following application of transverse orthopedic forces-a three-dimensional FEM study. Angle Orthod. 2003;73(1):12-20.

DOI:10.1043/00033219(2003)073<0012: SOSDAD>2.0.CO;2.

[13] Cantarella D, Dominguez-Mompell R, Moschik C, Mallya SM, Pan HC, Alkahtani MR, Elkenawy I, Moon W. Midfacial changes in the coronal plane induced by microimplant-supported skeletal expander, studied with cone-beam computed tomography images. Am J 
Orthod Dentofac Orthop. 2018;154(3): 337-45.

DOI:10.1016/j.ajodo.2017.11.033.

[14] Farhangfar A, Bogowicz P, Heo G, Lagravère $\mathrm{MO}$. Palatal bone resorption in bone-anchored maxillary expander treatment. Int Orthod. 2012;10(3):27488.

DOI:10.1016/j.ortho.2012.06.005.

[15] Lagravère MO, Gamble J, Major PW, Heo G. Transverse dental changes after tooth-borne and bone-borne maxillary expansión. Int Orthod. 2013;11(1):21-34.

DOI:10.1016/j.ortho.2012.12.003.

\section{CÓMO CITAR ESTE ARTÍCULO}

Rojas P, Córdova P, Santos G, Vergara C. Descripción de biomecánica asociada a disyunción maxilar con anclaje esqueletal: Reporte de caso. Appli Sci Dent. 2021:2(2); $32-41$

DOI: 10.22370/asd.2021.2.2.2595

Applied Sciences in Dentistry, revista científica de la Facultad de Odontología de la Universidad de Valparaíso, de Acceso Abierto y de Publicación Continua.

Son aceptados trabajos originales e inéditos, abarcando cartas al editor, comunicaciones breves, artículos de investigación, casos clínicos y revisiones bibliográficas.

Correo electrónico de contacto:

contacto.asdj@uv.cl

editor.asdj@uv.cl

Página Web:

https://revistas.uv.cl/index.php/asid

Redes Sociales

Instagram @asd.journal

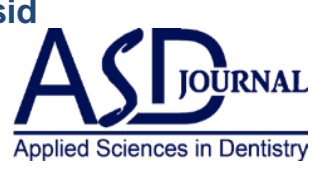

